\title{
Influence of Pecan Nut Pretreatment on the Physical Quality of Oil Bodies
}

\author{
Pujuan Zhang, ${ }^{1,2}$ Vincenzo Di Bari, ${ }^{2}$ Rhianna Briars, ${ }^{2}$ Zarani Mat Taher, ${ }^{2}$ Jiamiao Yuan, \\ Guangqin Liu, ${ }^{1}$ and David Gray ${ }^{2}$ \\ ${ }^{1}$ Institute of Horticulture, Jiangsu Academy of Agricultural Sciences, 50 Zhongling Street, Nanjing 210014, China \\ ${ }^{2}$ Division of Food Sciences, School of Biosciences, University of Nottingham, Sutton Bonington Campus, Loughborough LE12 5RD, UK
}

Correspondence should be addressed to Guangqin Liu; liuguangqin@126.com and David Gray; david.gray@nottingham.ac.uk

Received 21 January 2017; Accepted 19 March 2017; Published 18 May 2017

Academic Editor: Jorge Barros-Velázquez

Copyright (C) 2017 Pujuan Zhang et al. This is an open access article distributed under the Creative Commons Attribution License, which permits unrestricted use, distribution, and reproduction in any medium, provided the original work is properly cited.

\begin{abstract}
A supply of pure, intact oil bodies is essential for carrying out morphological and biochemical studies of these plant organelles and exploring their application. Preparation requires a carefully controlled breakage of plant cells, followed by separation of the oil bodies from cytoplasm and cell debris. This paper focuses on the recovery and characterisation of oil bodies from pecan nuts where no work has been published to date. The results showed that soaking softens the nut tissue and appears to reduce the damage to oil bodies during grinding and centrifugal force must be carefully selected to minimise oil bodies damage on recovery. A $24 \mathrm{~h}$ soaking time coupled with a $5500 \mathrm{RCF}$ recovery force allows for the recovery of intact pecan nut oil bodies.
\end{abstract}

\section{Introduction}

In many oil-rich seeds, as well as in nuts, the lipids are stored in the form of small-sized, discrete, spherical organelles called oil bodies [1-3]. Oil bodies, also known as lipid bodies, oleosomes and spherosomes, serve as energy stores to support active metabolism periods and are composed of a neutral lipid matrix core, consisting mainly of triglycerides (TAGs), surrounded by a monolayer of phospholipid embedded with some unique integral proteins: oleosin, the major protein, stereolosin, and caleolosin [4-6]. The proteins, together with the phospholipids (PL), stabilise the oil bodies and maintain them as small discrete entities via surface charge and steric hindrance [6]. Intact oil bodies, with their noncoalescing nature, high polyunsaturated fatty acids and antioxidants content [7], and good oxidative stability [8], have been exploited for food and biotechnological and pharmaceutical applications [9-14].

Reports on isolating oil bodies can be traced back to at least the 1970s. Oil bodies have been isolated from a range of seeds: soybean [15], sunflower seed [16], almond [17, 18], walnut [19] Arabidopsis [20], and maize germ [21]. Characterisation of oil bodies extracted from different seed (rapeseed, sesame, cotton, flax, and maize) and nuts (peanut) has shown that the size of these organelles always falls within a narrow range $(0.6-2.0 \mu \mathrm{m})$ with an isoelectric point from 5.7 to 6.6 [22]. Therefore, at neutral $\mathrm{pH}$ oil bodies tend to be negatively charged.

The process of oil body isolation can be divided into three steps: (1) the disruption of the seed matrix (via wetgrinding) to allow the release of oil bodies into an aqueous slurry; (2) filtration of the slurry to yield a "milk," that is, a suspension of oil bodies in an aqueous medium, with reduced seed particulate material; and (3) concentration of the oil bodies into a cream through the centrifugation of the milk. Ding et al. [23] recognized that the type of homogenizer, the force applied, and the treatment time are all crucial factors for obtaining intact oil bodies, and the optimum parameters may differ from sample to sample. Also, the centrifuge speed can affect the size distribution of the oil bodies, by either being insufficient to promote the creaming of very small oil bodies or being too high, leading to oil body coalescence as also suggested by [21]. Presoaking of the seeds has been used to increase the yield of extraction of oil bodies, but the length of soaking time differs between the seeds/nut type and between 
authors: for palm kernels a period of $72 \mathrm{~h}$ is needed [22], but safflower seeds only need to be soaked overnight [24]; Tzen et al. [22] soaked soybeans for $1 \mathrm{~h}$, while Chen et al. [25] soaked soybeans for $18 \mathrm{~h}$, and Iwanaga et al. [15] applied an overnight soak. No justification for the selected length of soaking time is often reported in the works leading to variations from author to author. Furthermore, reported centrifugal forces used to form the oil body-rich cream also differ significantly between research groups, ranging from 5.000 RCF [24] to 20.000 RCF [26].

Pecan nut [Carya illinoinensis] is one of the world's most popular tree nuts with a global production of 81052 metric tons shelled in 2013 (http://www.nutfruit.org/). Its total lipid content is $72 \%$, composed of approximately $6 \%$ total saturated fatty acids (16:0, 18:0, and 20:0), 41\% total monounsaturated fatty acids (18:1, 20:1), and 22\% total polyunsaturated fatty acids (18:2, 18:3). Opalescent pecan nuts, which occur when oil is released due to oil body damage, were studied by Wakeling et al. [27]. Their results showed that opalescence first becomes evident in kernels after mechanical cracking and increases further as processing continues. This indicates that the mechanical force experienced during nut cracking is causing some internal damage to the kernel; the presence free oil suggests that at least some of the oil bodies are ruptured and oil is released, which can make its way to cut surfaces. An insight into the structural differences between opalescent and nonopalescent pecan nut was offered by Wakeling et al. [28] by taking scanning electron microscopy (SEM) and transmission electron microscopy (TEM) images of pecan nut sections. In nonopalescent pecan nuts the oil bodies' structure is preserved; the visualisation of broken oil bodies in opalescent pecans suggests that the membrane of the lipid organelles had been damaged with subsequent release of oil leading to opalescence.

Despite having developed a structural understanding of the differences between the types of pecans, to date no paper has been published on the recovery and characterisation of pecan nut oil bodies. Therefore, the aim of this work was to develop a method to extract oil bodies from pecan nuts. The parameters investigated were the effect of nuts presoaking and centrifugation force on the final size of oil bodies. Furthermore, due to the reported sensitivity of pecan nut oil bodies to mechanical forces, our work aimed at characterising oil bodies extracted from opalescent and nonopalescent pecan nuts, where the former are nuts which have experienced mechanical damage. The particle size of recovered oil body emulsions was measured, and light microscopy images were obtained to evaluate the quality of the oil bodies.

\section{Materials and Methods}

2.1. Materials. Pecan nuts with shells were purchased from Whole Food Online and were used as a raw material for the aqueous extraction of oil bodies. The pecan nut kernels were obtained using a hand cracker to remove the shell. Care was taken to avoid mechanical damage to oil bodies during nut shelling; occurring mechanical damage was manifested as an opalescent effect on exposed surface.
All chemicals used in this study were of analytical grade or above purity and sourced from Sigma Chemical Company (St. Louis, MO) unless otherwise stated. Deionised water was obtained using a Nanopure Water System (Nanopure Infinity, Barnstead International, IA) and used to prepare all solutions and emulsions.

2.2. Oil Body Extraction from Nonopalescent Pecan Nut. Oil bodies were isolated using an aqueous extraction method employing a grinding medium of $0.1 \mathrm{M}$ Tris- $\mathrm{HCl} \mathrm{pH} 8.0$ containing $1 \mathrm{mM}$ EDTA and a washing buffer of $1 \mathrm{mM}$ $\mathrm{NaHCO}_{3}$ containing $1 \mathrm{mM}$ EDTA pH 8.0. Soaking time and centrifugal force were altered according to individual experimental parameters (Section Effect of Nut Soaking Time and Effect of Centrifugal Force).

The nonopalescent pecan nut kernels (bright white interior kernels) were initially broken into small pieces by hand and then soaked in deionised water $(1: 10 \mathrm{w} / \mathrm{v})$ at $4^{\circ} \mathrm{C}$, for a length of time dependent on the experimental parameters. Sodium azide $(0.02 \mathrm{M}, 0.05 \%$ volume of soaking water) was added to avoid microbial spoilage. The soaked pecan nuts were then ground in the grinding medium $(1: 10 \mathrm{w} / \mathrm{v}$ with two drops of $0.02 \mathrm{M}$ sodium azide) in a Kenwood blender (BL315) at full power for 2 minutes. After blending, the slurry was filtered through three layers of cheesecloth. The filtrate was then centrifuged (Beckman J2-21 centrifuge, fixed rotor JA10) for 20 minutes at $4^{\circ} \mathrm{C}$. The centrifugal force was varied with experiments. The resulting upper layer was collected as the crude oil bodies (COBs) for analysis. The COBs were then washed twice with washing buffer $(1: 4 \mathrm{w} / \mathrm{w})$ followed by two rinses with deionised water $(1: 4 \mathrm{w} / \mathrm{w})$, through resuspension (shake gently first and then vortex for 2 minutes at the low level) and centrifugation at 2885 RCF (JOUAN CR3i $4 \times$ $280 \mathrm{~mL}$ multifunction centrifuge) for 20 minutes at $4^{\circ} \mathrm{C}$ to remove extraneous proteins and contaminants. The resulting upper layer was collected as the washed oil bodies (WOBs) for analysis.

2.3. Effect of Nut Soaking Time. In order to evaluate the effect of soaking time on the size of the recovered oil bodies, pecan nut pieces were soaked in deionised water for $0 \mathrm{~h}, 2 \mathrm{~h}, 6 \mathrm{~h}$, $12 \mathrm{~h}, 24 \mathrm{~h}$, and $72 \mathrm{~h}$ at $4^{\circ} \mathrm{C}$. After blending and filtering, the filtrate was centrifuged at $5500 \mathrm{RCF}$ for 20 minutes at $4^{\circ} \mathrm{C}$.

2.4. Percentage of Weight Change of Pecan Nuts during Soaking. To quantify the water absorbed, the mass of pecan nuts was measured at different time points. To calculate the percentage weight change, we use the following equation:

$$
\begin{aligned}
& \text { Weight Change (\%) } \\
& =\left[\frac{(\text { weight of soaked nuts }- \text { weight of dry nuts })}{\text { weight of dry nuts }}\right] \\
& \quad * 100 \% .
\end{aligned}
$$

2.5. Conductivity of Soaking Water. The conductivity of soaking water at $4^{\circ} \mathrm{C}$ was directly measured using a conductivity 
meter (HI 9033 multirange conductivity meter, HANNA instruments, Romania).

2.6. Effect of Centrifugal Force. To evaluate the effect of the centrifugal force on oil bodies size, the pecan nut kernels were soaked in deionised water for 24 hours. After blending and filtering, the filtrate (i.e., the "milk") was transferred into four centrifuge tubes and centrifuged separately using different centrifugal forces: $3000 \mathrm{RCF}, 5500 \mathrm{RCF}, 7500 \mathrm{RCF}$, and $10000 \mathrm{RCF}$, for 20 minutes at $4^{\circ} \mathrm{C}$.

2.7. Emulsion Preparation for Physical Properties Characterisation. The oil bodies emulsions were prepared by mixing $1 \mathrm{~g}$ (wet weight) of COB or WOB cream with $9 \mathrm{~g}$ of deionised water using the Wheaton Potter Elvehjem Tissue Grinder (Safe-Grind $55 \mathrm{~mL}$, Fisher Scientific). The emulsions were then used directly for light microscopy visualisation and particle size analysis.

2.8. Oil Body Extraction from Opalescent Pecan Nut. Opalescent pecan nut kernels, characterised by a milky iridescence [27], were broken into small pieces by hand and then soaked in deionised water for $24 \mathrm{~h}(1: 10 \mathrm{w} / \mathrm{v})$ at $4^{\circ} \mathrm{C}$. After blending and filtering, the filtrate was then centrifuged at $5500 \mathrm{RCF}$ for 20 minutes at $4^{\circ} \mathrm{C}$ to recover the COBs. Washing to obtain the WOBs was done as referred in Oil Body Extraction from nonopalescent pecan nut.

2.9. Light Microscopy Visualisation of Oil Bodies. The microstructure of all oil body emulsions was investigated using light microscopy (EVOS inverted microscope, UK). Oil body emulsions were gently shaken before measurement to ensure homogeneity. A small drop of oil body emulsion was then placed on a glass slide, covered with a cover slide, and imaged immediately at a magnification of $40 x$.

2.10. Particle Size Analysis of Oil Body Emulsions. The volume weighted mean diameter $\left(D_{4,3}\right)$ and the surface weighted mean diameter $\left(D_{3,2}\right)$ are commonly used to measure particle sizes. $D_{4,3}$ is used throughout the paper as this parameter is sensitive to the presence of large particles, whereas $D_{3,2}$ is more sensitive to the presence of smaller particles [29].

The particle size distribution of oil body emulsions was measured using a laser diffraction particle size analyser (LS 13 320, Beckman Coulter, High Wycombe, UK). One drop of samples was mixed into $125 \mathrm{~mL}$ dispersing liquid and circulated inside the Universal Liquid Module. The diffraction data was analysed using the Fraunhofer diffraction model. The distribution curve and volume-mean diameter $\left(D_{4,3}\right)$ were measured; readings were taken in triplicate for each sample and averaged.

2.11. Statistical Analysis. Extraction experiments were performed in triplicate. Statistical analysis to evaluate significant differences was performed by one-way ANOVA $(P<0.05)$.

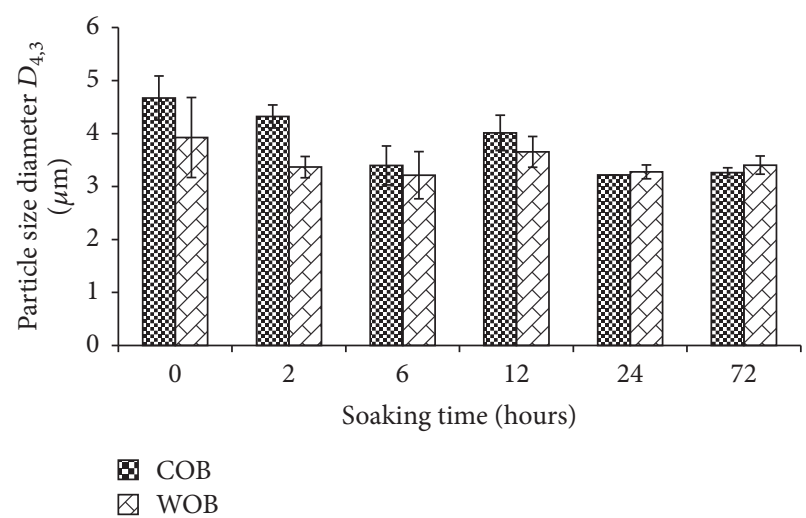

FIGURE 1: Average particle sizes of both crude oil body (COBs) and washed oil body (WOBs) emulsions at different seed presoaking times.

\section{Results and Discussions}

3.1. Effect of Presoaking on Physical Quality of Pecan Nut Oil Bodies. Tissue and cell disruption is required to release oil body organelles: grinding pecan nuts can damage the oil bodies, resulting in changes in their surface chemistry and size. It was hypothesised that nuts soaked prior to milling (presoaking) would soften the tissues and, therefore, minimise the mechanical forces experienced by the oil bodies during wet-milling and so reduce their damage. The influence of soaking times $(0 \mathrm{~h}, 2 \mathrm{~h}, 6 \mathrm{~h}, 12 \mathrm{~h}, 24 \mathrm{~h}$, and $72 \mathrm{~h})$ on the physical stability of recovered pecan nut oil bodies was investigated. The recovery force used for the purposes of this study was kept constant at $5500 \mathrm{RCF}$.

Results shown in Figure 1 suggest that there was a decrease in the average volume-mean diameter $\left(D_{4,3}\right)$ of $\mathrm{COB}$ with an increase in presoaking time. Crude oil bodies (COBs) from nonpresoaked $(0 \mathrm{~h})$ pecan nuts had the largest $D_{4,3}(4.70 \pm$ $0.42 \mu \mathrm{m}$ ), whilst the COBs isolated from $24 \mathrm{~h}$ and $72 \mathrm{~h}$ presoaked nuts had the smallest diameter $(3.20 \pm 0.002 \mu \mathrm{m}$ and $3.30 \pm 0.09 \mu \mathrm{m}$, resp.). This indicates that the plasticisation of the nut through presoaking in water reduces the damage caused to oil bodies during nut-tissue grinding. The particle size distributions of COBs confirm this result (Figure 2). For $0 \mathrm{~h}, 2 \mathrm{~h}, 6 \mathrm{~h}$, and $12 \mathrm{~h}$ soaking time distributions, two modes exist with one observed around $20 \mu \mathrm{m}$ suggesting the existence of large droplets or the presence of droplets aggregates, while for the $24 \mathrm{~h}$ and $72 \mathrm{~h}$ distributions there was just one mode around $3 \mu \mathrm{m}$ (Figure 2(a)). Light microscopy images support the results of the particle size analysis and distributions, as they clearly show large droplets of $\mathrm{COB}$ when the nuts were not soaked, while the COBs extracted from $24 \mathrm{~h}$ soaked nuts were smaller (Figure 3 ). These results suggest that the presoaking step has a direct impact on the size of the oil bodies and long soaking periods ( $\geq 24 \mathrm{~h}$ ) may be preferred. It should be pointed out here that no data are available in literature on pecan nut oil bodies. From published SEM/TEM images of nonopalescent pecan nut ultrastructure [28], we could estimate that the approximate particle size for pecan nut oil bodies ranges between 1 and $6 \mu \mathrm{m}$, which is above the 


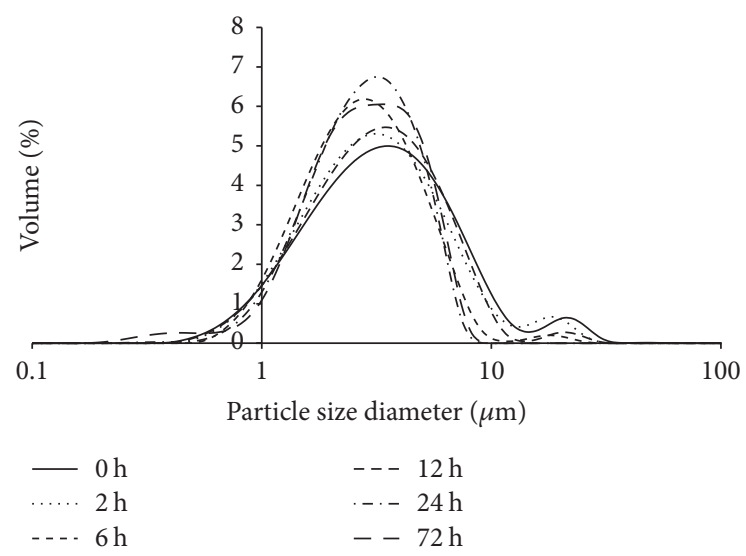

(a)

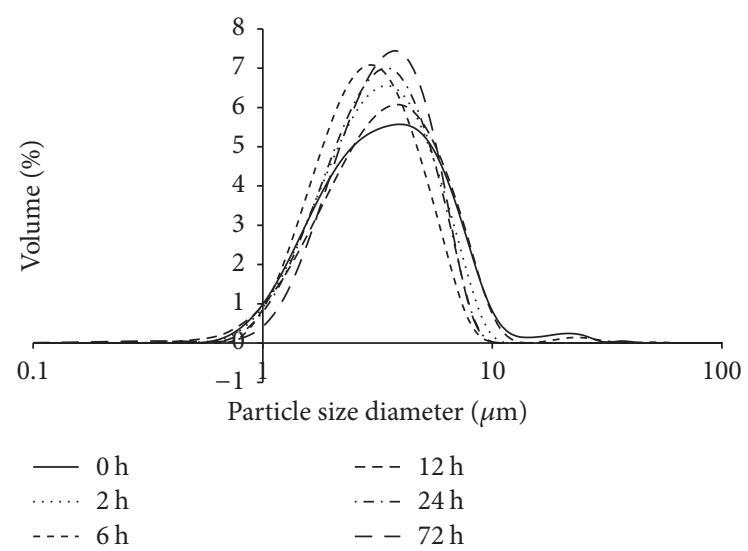

(b)

FIGURE 2: Particle size distributions of (a) crude oil body (COBs) and (b) washed oil body (WOBs) emulsions at different seed presoaking times.

common range reported in literature for oil bodies extracted from oil-rich seeds $(0.5-2.5 \mu \mathrm{m})[22,30]$.

The average $D_{4,3}$ of washed oil bodies (WOBs) was comparable across all oil body emulsions independently from the length of soaking (Figure 1). The size distribution curves for WOBs (Figure 2(b)) appear narrower compared to those obtained for the COBs, which indicates that the washing step is removing some of the larger droplets. We hypothesise that the largest droplets (above $15 \mu \mathrm{m}$, Figures 3(a) and 3(b)) may be coalesced oil bodies formed on grinding and weakly stabilised by interfacially active materials (proteins, micron size particle material, and phospholipids) released and/or produced on pecans milling. This interfacial material could be easily removed on COBs washing, which would result in the destabilisation and loss of those large droplets in the form of little amount of free oil. This hypothesis is supported by the absence of the peak at approximately $20 \mu \mathrm{m}$ (Figure 2(b)) and of the majority of large droplets in washed oil body emulsions produced following $0 \mathrm{~h}$ of soaking (Figure 4).

A better understanding of the effect of soaking on oil bodies' size was gained by water uptake and conductivity measurements over time (Figure 5). The percentage weight change of soaked pecan nuts increased dramatically from
$2 \mathrm{~h}$ to $6 \mathrm{~h}$ and from $22 \%$ to $33 \%$ and continued to increase from $6 \mathrm{~h}$ until $24 \mathrm{~h}$, although to a smaller extent (from $33 \%$ to $37 \%)$. After $24 \mathrm{~h}$ no significant increase occurred. This result suggests that if the soaking time is below $24 \mathrm{~h}$, the nuts still have the capability to absorb water and might still be hard on grinding.

Another approach to evaluate the evolution of pecan nut over soaking is by measurement of the electrical conductivity of the soaking water. The conductivity test is acknowledged as suitable method to evaluate the loss of cell membrane integrity through measuring the concentration of electrolytes released by seeds during imbibition [31-33]. The deterioration of the cell membranes during presoaking may facilitate the release of oil bodies from the nut tissue. In line with the data of water mass uptake (Figure 5), water conductivity continues to increase until $24 \mathrm{~h}$ of soaking, that is, until the net water uptake is zero. If membrane disruption and subsequent conductivity increase are caused by enzymatic activity, then conductivity may be an indirect measure of waterinduced enzyme activation in the seed. The risk associated with prolonged soaking times is that the activated enzymes may start destabilising the oil bodies to produce the energy needed on germination. Nevertheless, in this study the size of oil bodies is unaffected by prolonged soaking time $(72 \mathrm{~h})$.

The results from the presoaking experiment strongly suggest that softer nuts yield fewer damaged oil bodies. With increasing soaking time, the plasticisation of the nuts should increase, and consequently the mechanical forces experienced by the oil bodies during grinding should decrease. This would ultimately result in less damaged oil bodies with a narrower size distribution as observed for soaking times above $24 \mathrm{~h}$. In order to understand in more depth the effect of nut plasticisation on oil bodies' size, it would be important to measure the mechanical properties of pecan nuts during soaking. An attempt was made to study pecans mechanical properties; nevertheless, the irregular shape of pecan nuts presents a challenge to these measurements and these aspects may be investigated in the future.

\subsection{Effect of Centrifugal Force on Physical Quality of Pecan Nut} Oil Bodies. The purpose of this series of experiments was to determine the influence of centrifugal force on the physical stability of pecan nut oil bodies. The recovery forces studied were $3000 \mathrm{RCF}, 5500 \mathrm{RCF}, 7500 \mathrm{RCF}$, and 10,000 RCF, and all pecan nuts were presoaked for $24 \mathrm{~h}$ to minimise damage experiences during grinding.

The average diameter $\left(D_{4,3}\right)$ of COBs was approximately $3.3 \mu \mathrm{m}$ when recovery forces of $3000 \mathrm{RCF}, 5500 \mathrm{RCF}$, and 7500 RCF were used (Figure 6), which is similar to the size of pecan nut oil bodies in vivo, as estimated through SEM/TEM images of pecan nuts from literature [28]. When the recovery force was increased to 10,000 RCF, significant differences $(P<$ 0.05 ) were observed as $D_{4,3}$ of COBs increased to $4.8 \mu \mathrm{m}$ (Figure 6). This suggests that, due to high centrifugal force, oil bodies may aggregate and eventually coalesce during the centrifugation process. The particle size distributions of COBs (Figure 7(a)) also indicate the appearance of larger droplets when the $10,000 \mathrm{RCF}$ recovery force was used: the 


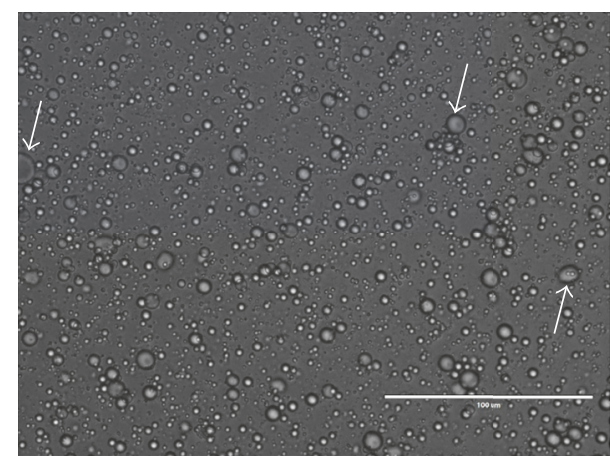

(a)

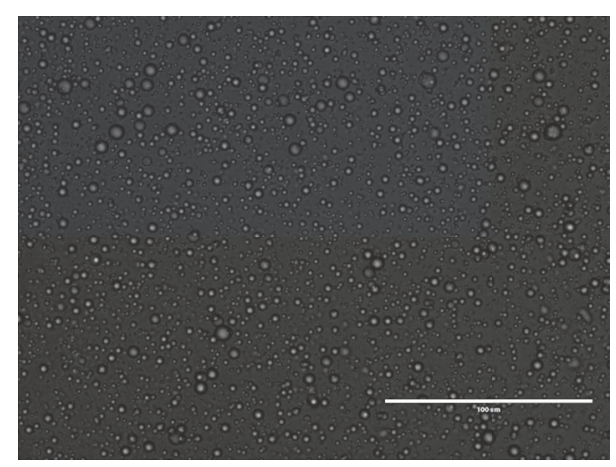

(c)

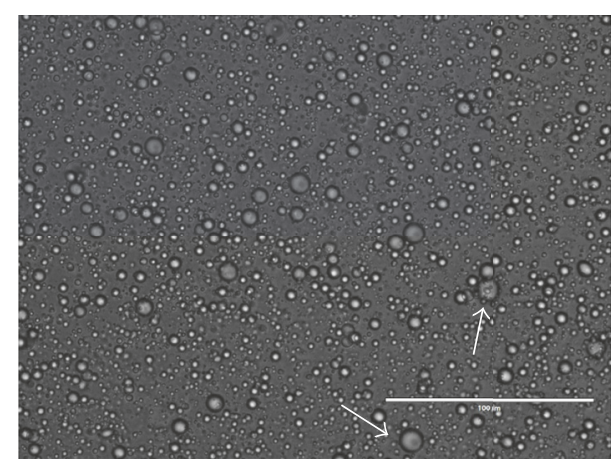

(b)

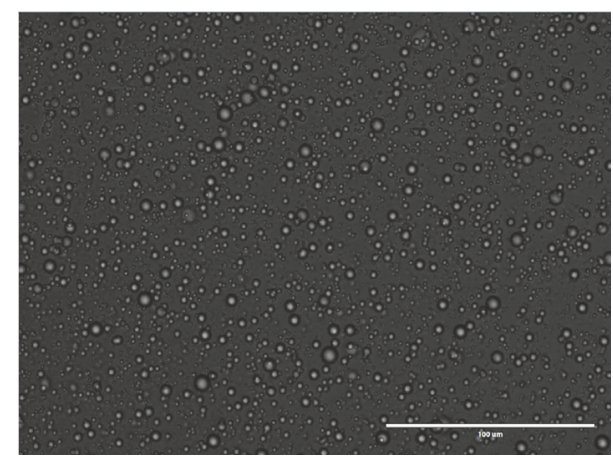

(d)

FIGURE 3: Light micrographs of crude oil body (COBs) emulsions of pecan nuts soaked for $0 \mathrm{~h}(\mathrm{a}$ and $\mathrm{b}$ ) and $24 \mathrm{~h}$ ( $\mathrm{c}$ and $\mathrm{d}$ ). The scale bars represent $100 \mu \mathrm{m}$.

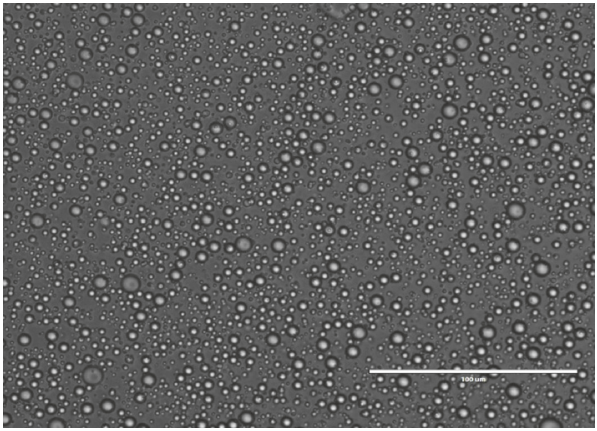

(a)

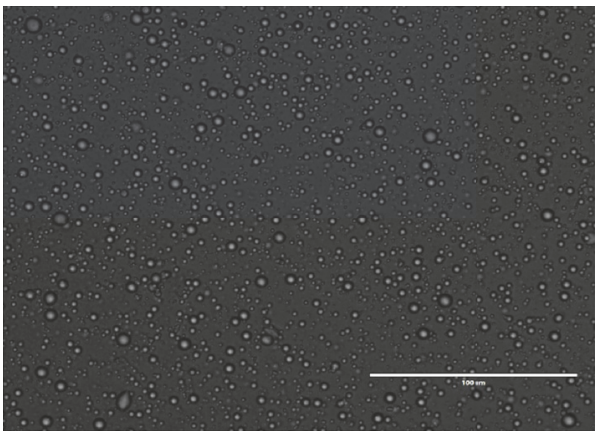

(c)

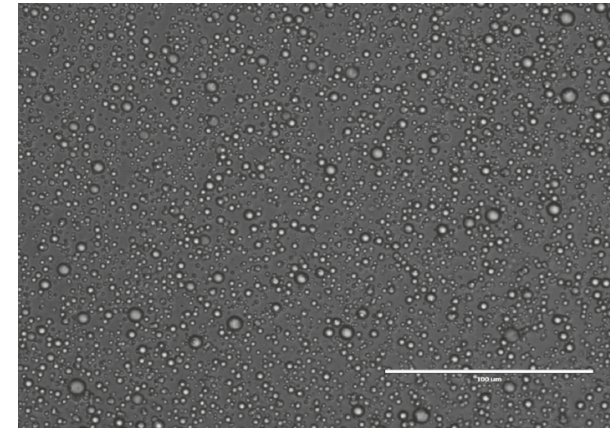

(b)

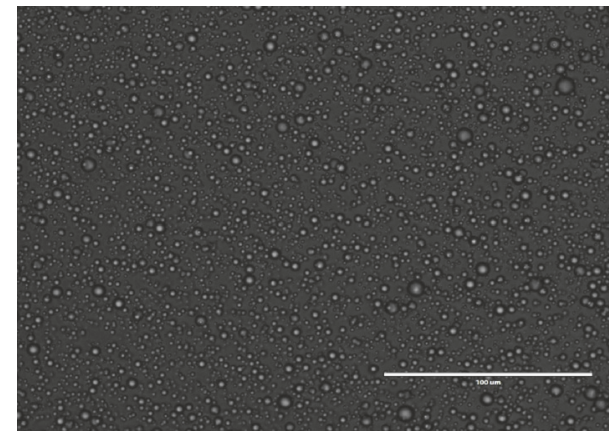

(d)

FigURE 4: Light micrographs of washed oil body (WOBs) emulsions of pecan nuts soaked for $0 \mathrm{~h}$ ( $\mathrm{a}$ and $\mathrm{b}$ ) and $24 \mathrm{~h}$ ( $\mathrm{c}$ and d). The scale bars represent $100 \mu \mathrm{m}$. 


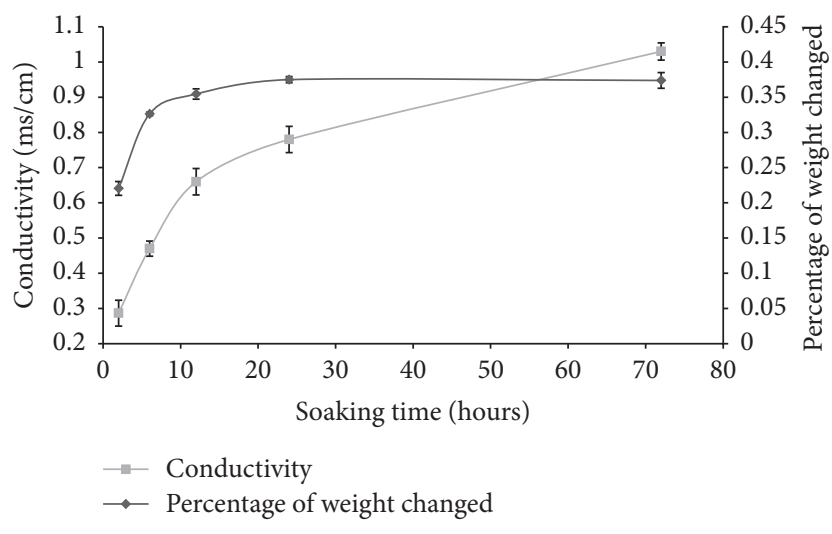

FIGURE 5: Percentage of weight changed on soaking and conductivity of soaking water.

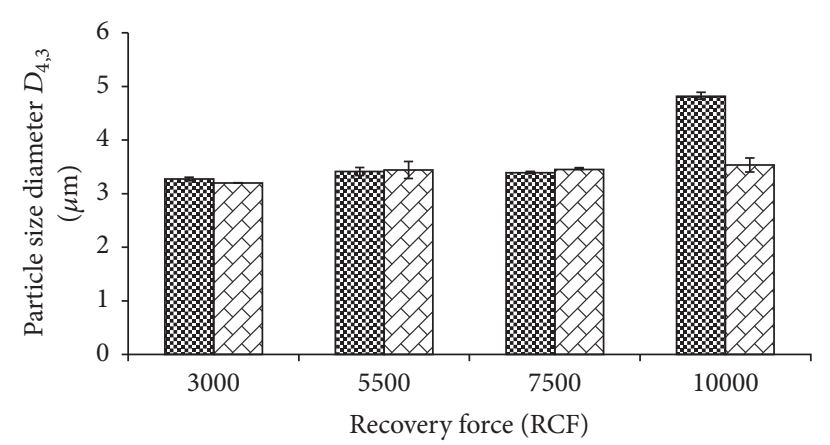

田OB

$8 \mathrm{WOB}$

Figure 6: Average particle sizes of both crude oil body (COBs) and washed oil body (WOBs) emulsions obtained using different centrifugal forces.

size distribution curve produced using the highest recovery force is broader than the distribution curves obtained at the other three centrifugal forces. Furthermore, for samples recovered at 10,000 RCF another mode appeared at $25 \mu \mathrm{m}$ (Figure 7(a)), which indicates the presence of large droplets as also shown in Figure 8(b), where many large oil bodies and oil bodies aggregates are observed. When the recovery force was lower than 10,000 RCF, the oil bodies appear as small and discrete droplets (Figure 8(a)).

$10,000 \mathrm{RCF}$ is a commonly reported recovery force in literature for oil bodies separation [3, 34, 35]. However, results obtained in this study show that the pecan nut oil bodies were damaged at this force. One possible explanation for the damage caused by the highest RCF force might be that the membrane tension of pecan nut oil bodies is low due to their relatively large size: since membrane tension decreases with increasing particle size [36], the pecan nut oil bodies resistance to an externally applied force is likely to be lower than that of oil bodies extracted from other lipid sources.

3.3. Oil Body Extraction from Opalescent Pecan Nuts. Opalescent pecan nuts are a type of pecan nut where at least some

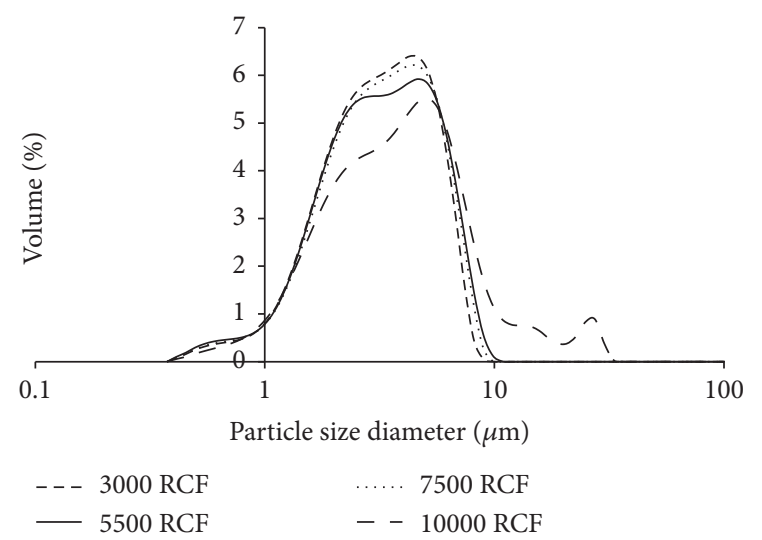

(a)

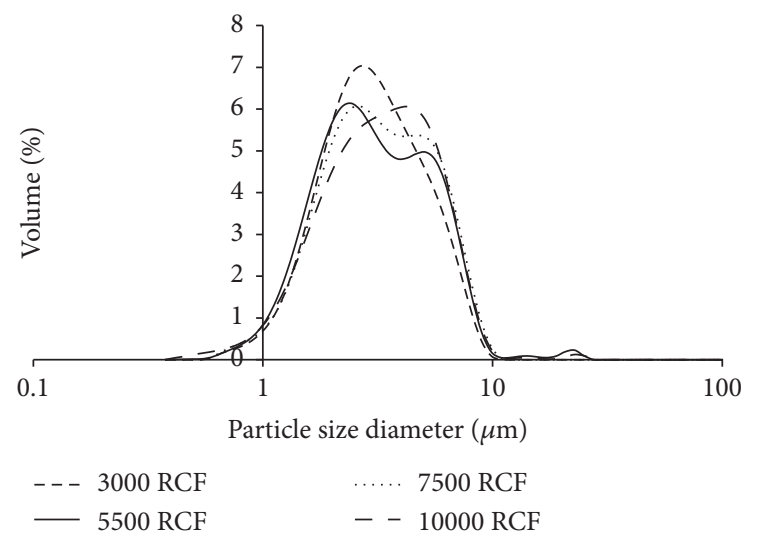

(b)

FIGURE 7: Particle size distributions of (a) crude oil body (COBs) and (b) washed oil body (WOBs) emulsions obtained using different recovery forces.

of the oil bodies have been broken inside the nut and, due to the oil release, the kernel exhibits a brown or caramel colour compared with the white interior of nonopalescent pecan nuts (Figure 9). The appearance of opalescence in nuts we provided in our work is in line with the current (fairly limited) literature on nuts, where the phenomenon of opalescence is considered a result simply of mechanical damage occurring at various processing stages. However, it is very likely that mechanical damage of the OBs, even if occurred at a limited extent, may cause the triggering of native enzymes and their activity would further promote OBs degradation leading to oil leak and opalescence. The opalescent pecan nuts (selected out from shelled pecan nuts) were presoaked for $24 \mathrm{~h}$ and the oil bodies recovered using 5500 RCF. These conditions were selected since they limit the mechanical damage of the oil bodies. $D_{4,3}$ values of both COBs $(5.7 \mu \mathrm{m})$ and WOBs $(5.2 \mu \mathrm{m})$ extracted from opalescent pecan nuts are larger than those for the nonopalescent pecan nuts $\left(D_{4,3}\right.$ of $3.2 \mu \mathrm{m}$ and $3.3 \mu \mathrm{m}$ for COBs and WOBs, resp.) (Figure 10). The particle size distributions of opalescent pecan nuts were wider than that of the oil bodies from nonopalescent nuts and were clearly shifted to the right (Figure 11). Many large droplets were visible in light microscopy images for both the COBs 


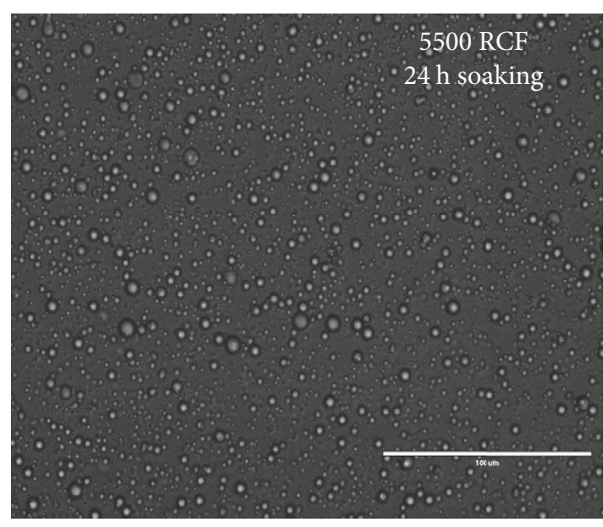

(a)

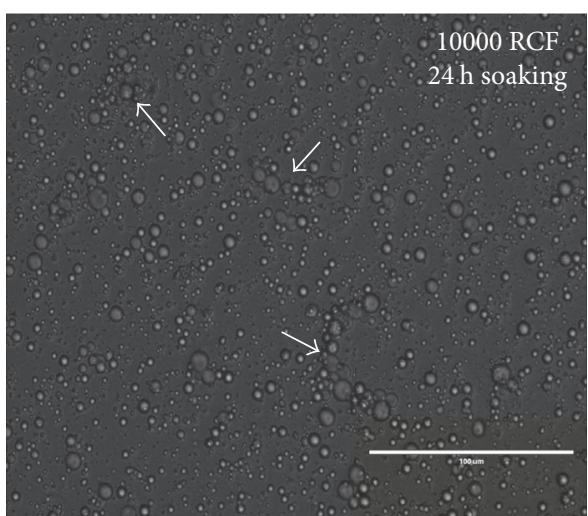

(b)

FIGURE 8: Light micrographs of crude oil body (COBs) emulsions of pecan nuts recovered using (a) 5500 RCF and (b) 10000 RCF. The arrows indicate aggregations. The scale bars represent $100 \mu \mathrm{m}$.

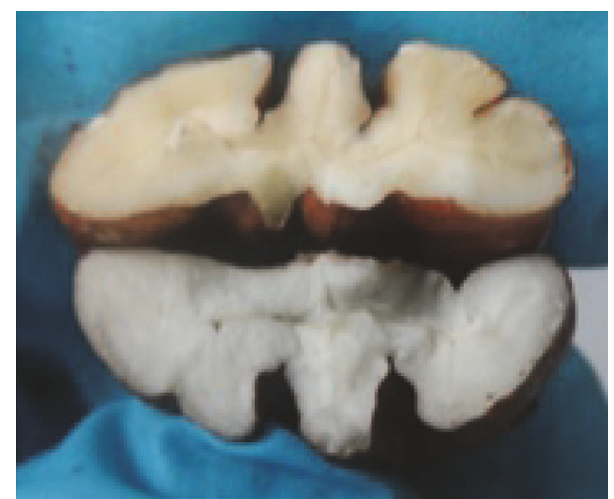

FIGURE 9: Image showing the contrast between (top) opalescent and (bottom) nonopalescent pecan nuts.

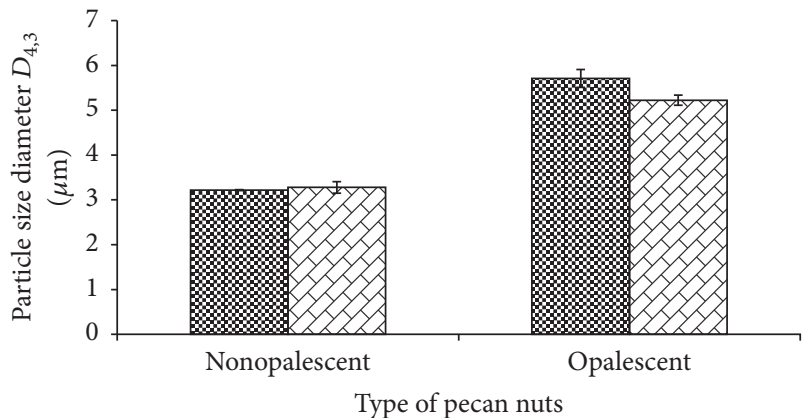

$\$ \mathrm{COB}$

WOB

FIGURE 10: Average particle sizes of both crude oil body (COBs) and washed oil body (WOBs) emulsions of opalescent and nonopalescent pecan nuts.

and WOBs of opalescent pecan nuts (Figure 12). The presence of large droplets in COBs suggests that mechanical damage of oil bodies had occurred prior to wet-milling producing the opalescence effect. This result is in agreement with results

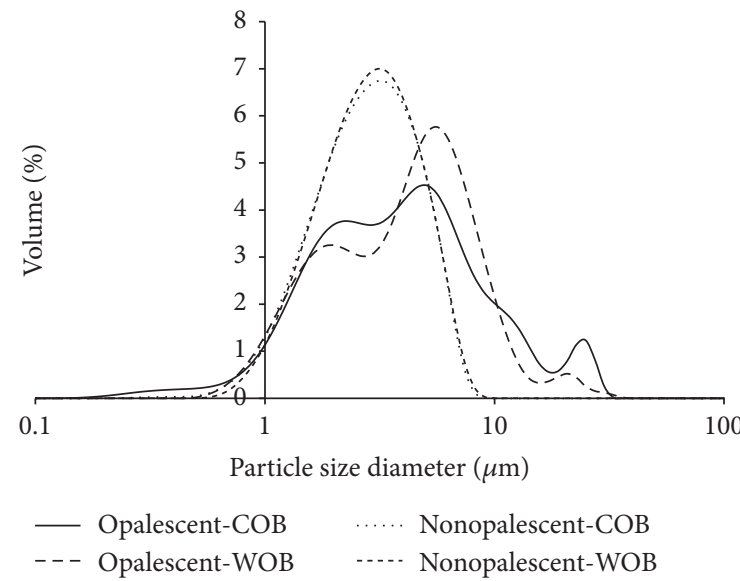

FIGURE 11: Particle size distributions of crude oil body (COBs) and washed oil body (WOBs) emulsions of opalescent and nonopalescent pecan nuts.

from Wakeling et al. [27, 28], who demonstrated that the opalescence results from the release of oil from broken oil bodies.

On washing no change in the size distribution and appearance of oil bodies can be observed (Figures 11 and 12). This result remains unexplained. Considering that the large oil bodies observed for opalescent pecans are able to endure the washing step, it could be hypothesised that these oil bodies have a different surface chemistry compared to the large droplets formed on grinding of nonopalescent nuts after limited soaking (less than $6 \mathrm{~h}$ ).

It has been reported in literature that pecans grown in the United States developed less severe opalescence than those grown in Australia because these pecans had lower levels of calcium, coincident with higher levels of oil [27]. Pecan composition varies depending on location, climate condition, horticultural practices, cultivar, season, and maturity level [37]; hence, it is important to establish the optimum soaking 


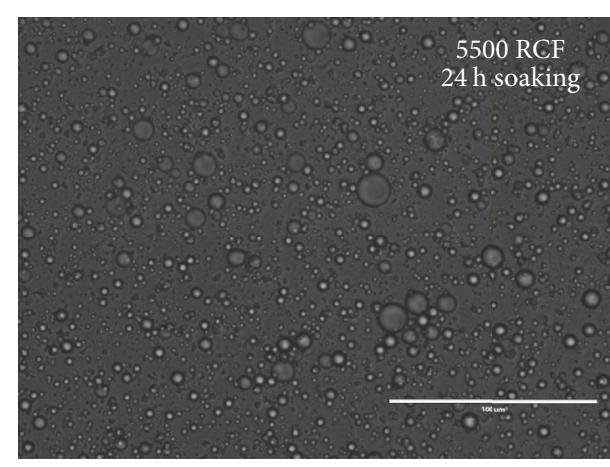

(a)

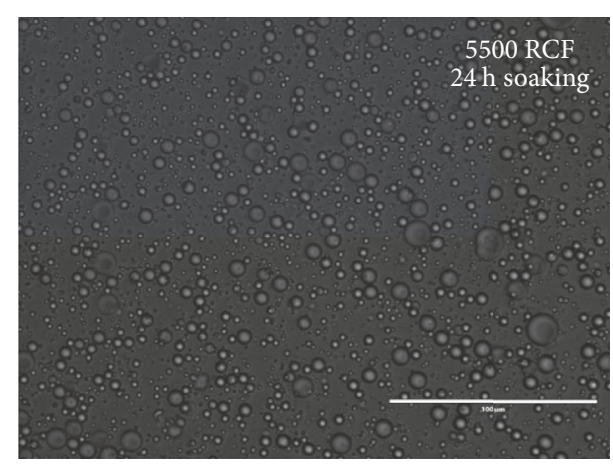

(b)

FIGURE 12: Light micrographs of (a) crude oil body (COB) and (b) washed oil body (WOB) emulsions of opalescent pecan nuts.

time and centrifugal force when different pecan nut cultivars are being used for oil bodies extraction.

\section{Conclusions}

This work is the first to describe a method for the extraction and characterisation of pecan nut oil bodies. It was demonstrated that presoaking affects the physical properties of pecan nut oil bodies. Through the soaking experiment we found that there was a decrease in volume-mean diameter $\left(D_{4,3}\right)$ of COBs with an increase in presoaking time suggesting that the plasticisation during soaking in water reduces oil bodies damage following grinding. The smallest oil bodies were extracted from nuts presoaked for $24 \mathrm{~h}$ and $72 \mathrm{~h}$, and the size of the isolated lipid organelles was similar to that of pecan nut oil bodies in vivo. We also show that the washing step is removing the large droplets and giving narrower distribution curves compared to the COBs only when mechanical damage of the oil bodies has occurred. Evaluation of the effect of the centrifugal force showed that using 10,000 RCF recovery force induces damaging of the oil bodies with an increase of $D_{4,3}$ of COBs from 3.3 to $4.8 \mu \mathrm{m}$. Finally, we have provided a quantitative difference of the COBs and WOBs size recovered from opalescent and nonopalescent pecan nuts: oil bodies extracted from opalescent pecans are larger than those recovered from nonopalescent nuts, confirming that the oil bodies inside the opalescent pecan nut are damaged prior to wet-milling. From all of our findings, we can conclude that a $24 \mathrm{~h}$ soaking time coupled with a recovery force of $5500 \mathrm{RCF}$ allows for the recovery of intact pecan nut oil bodies.

\section{Additional Points}

Intact oil bodies have been exploited for food and biotechnological and pharmaceutical applications because of their noncoalescing nature, high polyunsaturated fatty acids, antioxidants content, and good oxidative stability. The oil content of pecan nuts is around $70 \%$ and rich in unsaturated fatty acids. The method of isolating intact pecan nut oil bodies can promote the application of pecan nut oil bodies and the discussed recovery factors include soaking time and recovery force will give a reference for the oil body isolation from other materials.

\section{Conflicts of Interest}

The authors declare that they have no conflicts of interest.

\section{Acknowledgments}

The authors wish to thank Guy Channell and Bettina Wolf for providing suggestions and data analysis. This research was supported by grants from the Jiangsu Provincial Program for Forestry (Grant no. lysx (2013) 06) and the China Special Fund for Forest Scientific Research in the Public Welfare (Grant no. 201204404).

\section{References}

[1] L. Y. Yatsu and T. J. Jacks, "Spherosome Membranes: Half UnitMembranes," Plant Physiology, vol. 49, no. 6, pp. 937-943, 1972.

[2] A. H. Huang, "Lipid bodies," in Cell Components, vol. 1 of Modern Methods of Plant Analysis, pp. 145-151, Springer, Berlin, Germany, 1985.

[3] J. T. C. Tzen, G. C. Lie, and A. H. C. Huang, "Characterization of the charged components and their topology on the surface of plant seed oil bodies," Journal of Biological Chemistry, vol. 267, no. 22, pp. 15626-15634, 1992.

[4] A. H. C. Huang, "Oil bodies and oleosins in seeds," Annual Review of Plant Physiology and Plant Molecular Biology, vol. 43, no. 1, pp. 177-200, 1992.

[5] A. H. C. Huang, "Oleosins and oil bodies in seeds and other organs," Plant Physiology, vol. 110, no. 4, pp. 1055-1061, 1996.

[6] J. T. Tzen, "Integral Proteins in Plant Oil Bodies," ISRN Botany, vol. 2012, Article ID 173954, 16 pages, 2012.

[7] D. A. White, I. D. Fisk, S. Makkhun, and D. A. Gray, "In vitro assessment of the bioaccessibility of tocopherol and fatty acids from sunflower seed oil bodies," Journal of Agricultural and Food Chemistry, vol. 57, no. 13, pp. 5720-5726, 2009.

[8] O. A. Karkani, N. Nenadis, C. V. Nikiforidis, and V. Kiosseoglou, "Effect of recovery methods on the oxidative and physical stability of oil body emulsions," Food Chemistry, vol. 139, no. 1-4, pp. 640-648, 2013. 
[9] D. A. White, I. D. Fisk, J. R. Mitchell, B. Wolf, S. E. Hill, and D. A. Gray, "Sunflower-seed oil body emulsions: rheology and stability assessment of a natural emulsion," Food Hydrocolloids, vol. 22, no. 7, pp. 1224-1232, 2008.

[10] S. C. Bhatla, V. Kaushik, and M. K. Yadav, "Use of oil bodies and oleosins in recombinant protein production and other biotechnological applications," Biotechnology Advances, vol. 28, no. 3, pp. 293-300, 2010.

[11] G. G. Adams, S. Imran, S. Wang et al., "Extraction, isolation and characterisation of oil bodies from pumpkin seeds for therapeutic use," Food Chemistry, vol. 134, no. 4, pp. 1919-1925, 2012.

[12] H. M. Deckers, G. Van Rooijen, J. Boothe, J. Goll, and M. M. Moloney, "Oil body based personal care products," In Google Patents, 2001.

[13] H. Deckers, G. Rooijen, J. Boothe et al., "Immunogenic formulations comprising oil bodies," In Google Patents, 2005.

[14] H. M. Deckers, G. Van Rooijen, J. Boothe, J. Goll, and M. M. Moloney, "Products for topical applications comprising oil bodies," In Google Patents, 2003.

[15] D. Iwanaga, D. A. Gray, I. D. Fisk, E. A. Decker, J. Weiss, and D. J. McClements, "Extraction and characterization of oil bodies from soy beans: a natural source of pre-emulsified soybean oil," Journal of Agricultural and Food Chemistry, vol. 55, no. 21, pp. 8711-8716, 2007.

[16] I. D. Fisk, D. A. White, M. Lad, and D. A. Gray, "Oxidative stability of sunflower oil bodies," European Journal of Lipid Science and Technology, vol. 110, no. 10, pp. 962-968, 2008.

[17] F. Beisson, N. Ferté, S. Bruley, R. Voultoury, R. Verger, and V. Arondel, "Oil-bodies as substrates for lipolytic enzymes," Biochimica et Biophysica Acta - Molecular and Cell Biology of Lipids, vol. 1531, no. 1-2, pp. 47-58, 2001.

[18] S. Gallier, K. C. Gordon, and H. Singh, "Chemical and structural characterisation of almond oil bodies and bovine milk fat globules," Food Chemistry, vol. 132, no. 4, pp. 1996-2006, 2012.

[19] P. Jolivet, C. Deruyffelaere, C. Boulard et al., "Deciphering the structural organization of the oil bodies in the Brassica napus seed as a mean to improve the oil extraction yield," Industrial Crops and Products, vol. 44, pp. 549-557, 2013.

[20] P. Jolivet, E. Roux, S. D’Andrea et al., "Protein composition of oil bodies in Arabidopsis thaliana ecotype WS," Plant Physiology and Biochemistry, vol. 42, no. 6, pp. 501-509, 2004.

[21] C. V. Nikiforidis and V. Kiosseoglou, "Aqueous extraction of oil bodies from maize germ (Zea mays) and characterization of the resulting natural oil-in-water emulsion," Journal of Agricultural and Food Chemistry, vol. 57, no. 12, pp. 5591-5596, 2009.

[22] J. T. C. Tzen, Y.-Z. Cao, P. Laurent, C. Ratnayake, and A. H. C. Huang, "Lipids, proteins, and structure of seed oil bodies from diverse species," Plant Physiology, vol. 101, no. 1, pp. 267-276, 1993.

[23] Y. Ding, S. Zhang, L. Yang et al., "Isolating lipid droplets from multiple species," Nature Protocols, vol. 8, no. 1, pp. 43-51, 2013.

[24] D. J. Lacey, N. Wellner, F. Beaudoin, J. A. Napier, and P. R. Shewry, "Secondary structure of oleosins in oil bodies isolated from seeds of safflower (Carthamus tinctorius L.) and sunflower (Helianthus annuus L.)," Biochemical Journal, vol. 334, no. 2, pp. 469-477, 1998.

[25] Y. Chen, L. Zhao, Y. Cao, X. Kong, and Y. Hua, "Oleosins (24 and $18 \mathrm{kDa}$ ) are hydrolyzed not only in extracted soybean oil bodies but also in soybean germination," Journal of Agricultural and Food Chemistry, vol. 62, no. 4, pp. 956-965, 2014.
[26] C. V. Nikiforidis, V. Kiosseoglou, and E. Scholten, "Oil bodies: an insight on their microstructure - maize germ vs sunflower seed," Food Research International, vol. 52, no. 1, pp. 136-141, 2013.

[27] L. T. Wakeling, R. L. Mason, B. R. D’Arcy, and N. A. Caffin, "Opalescence in Australian-grown pecan kernels: occurrence and causes," Journal of Food Science, vol. 67, no. 8, pp. 28732880,2002

[28] L. T. Wakeling, R. L. Mason, B. R. D’Arcy, N. A. Caffin, and D. Gowanlock, "Microscopic structure of opalescent and nonopalescent pecans," Journal of Food Science, vol. 68, no. 7, pp. 2238-2242, 2003.

[29] S. Couvreur and C. Hurtaud, "Globule milk fat: secretion, composition, function and variation factors," INRA Productions Animales, vol. 20, no. 5, pp. 369-382, 2007.

[30] J. T. Tzen and A. Huang, "Surface structure and properties of plant seed oil bodies," The Journal of Cell Biology, vol. 117, no. 2, pp. 327-335, 1992.

[31] M. V. Miguel and J. Marcos Filho, "Potassium leakage and maize seed physiological potential," Scientia Agricola, vol. 59, no. 2, pp. 315-319, 2002.

[32] M. Panobianco, R. D. Vieira, and D. Perecin, "Electrical conductivity as an indicator of pea seed aging of stored at different temperatures," Scientia Agricola, vol. 64, no. 2, pp. 119-124, 2007.

[33] R. D. Vieira, D. M. TeKrony, D. B. Egli, W. P. Bruenning, and M. Panobianco, "Temperature during soybean seed storage and the amount of electrolytes of soaked seeds solution," Scientia Agricola, vol. 65, no. 5, pp. 496-501, 2008.

[34] D. A. White, I. D. Fisk, and D. A. Gray, "Characterisation of oat (Avena sativa L.) oil bodies and intrinsically associated Evitamers," Journal of Cereal Science, vol. 43, no. 2, pp. 244-249, 2006.

[35] R. L. C. Chuang, J. C. F. Chen, J. Chu, and J. T. C. Tzen, "Characterization of seed oil bodies and their surface oleosin isoforms from rice embryos," Journal of Biochemistry, vol. 120, no. 1, pp. 74-81, 1996.

[36] C.-C. Peng, I.-P. Lin, C.-K. Lin, and J. T. C. Tzen, "Size and stability of reconstituted sesame oil bodies," Biotechnology Progress, vol. 19, no. 5, pp. 1623-1626, 2003.

[37] E. K. Heaton, A. Shewfelt, A. Badenhop, and L. Beuchat, Pecan, Handling, Storage, Processing and Utilization, Research BulletinGeorgia Experiment Station, Ga, USA, 1977. 

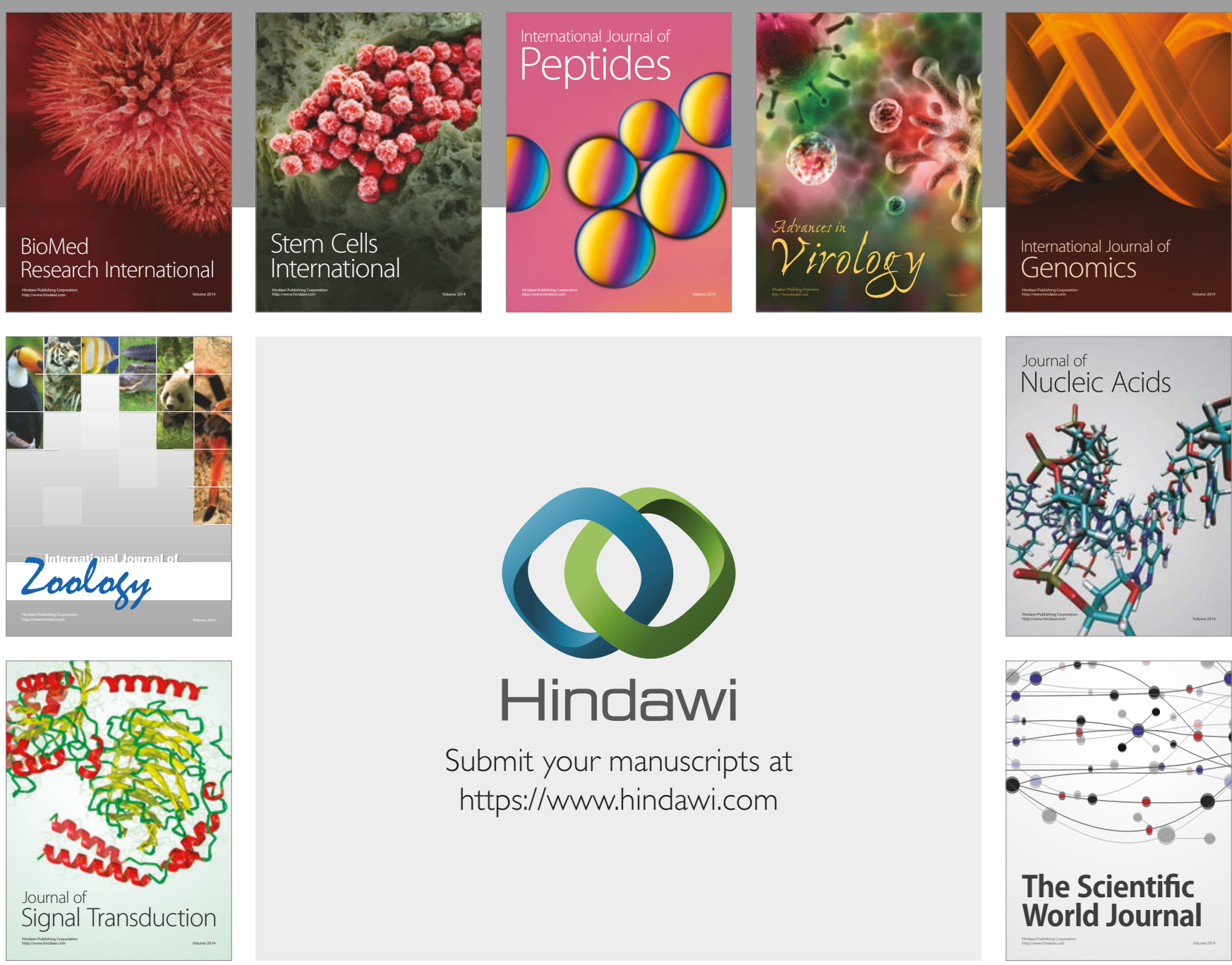

Submit your manuscripts at

https://www.hindawi.com
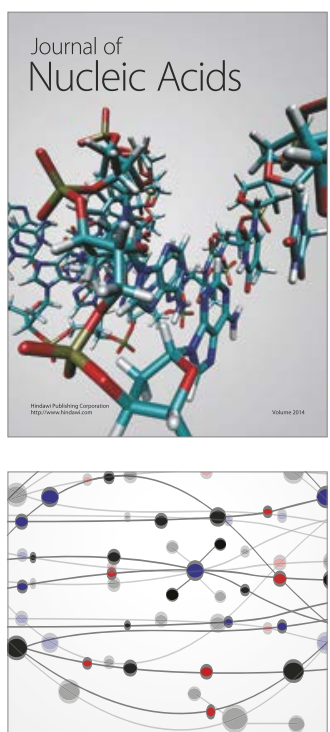

The Scientific World Journal

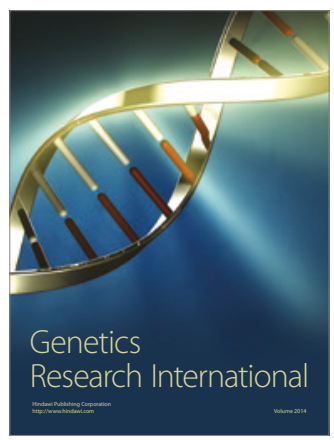

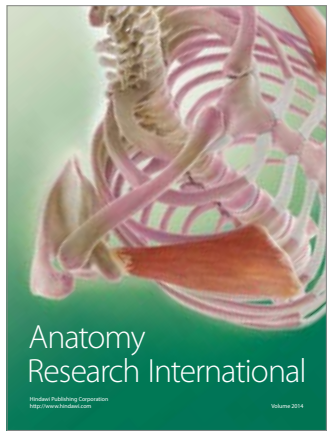

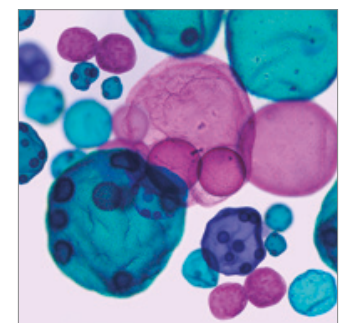

International Journal of Microbiology
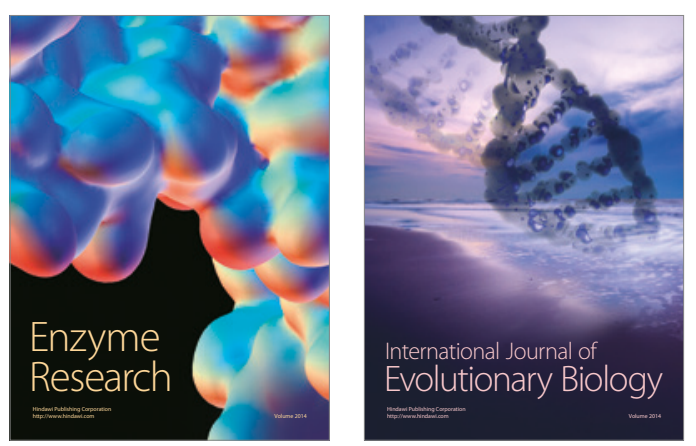
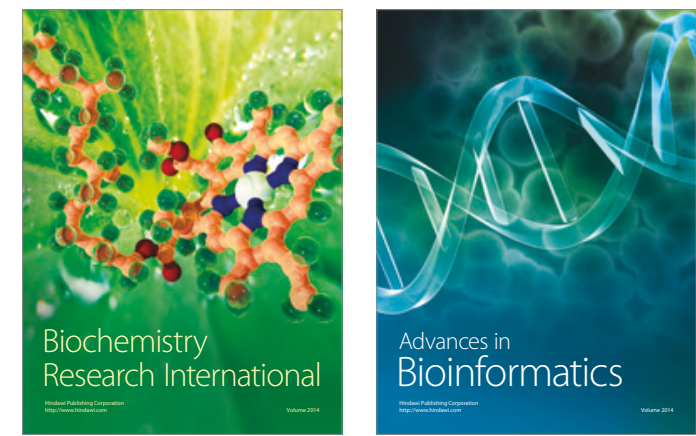

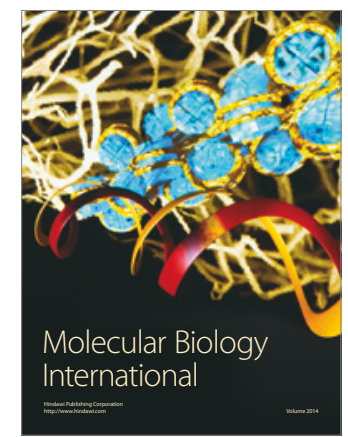

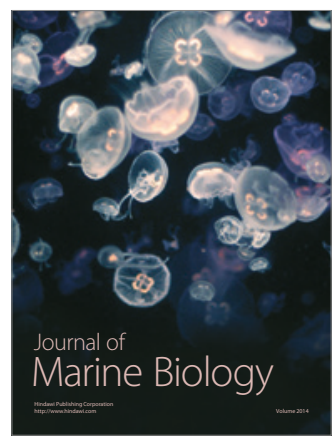

